\title{
A etnobotânica aplicada à úlcera gástrica e avaliação farmacológica de Solanum stipulaceum
}

\author{
Cirlane Alves Araújo de Limaa, Denise Maria da Silvaa, Evlyn Larisse da Silva Vilara, Marcos \\ Oliveira Rochab, Washington Soares Ferreira-Júniorc, Josemar Sena Batistad, Maria Silene da \\ Silvaa,e $\mathrm{e}^{*}$ \\ ${ }^{a}$ Grupo de pesquisa em etnobotânica e etnofarmacologia, Universidade Estadual de Alagoas, Arapiraca, 57306-120,Brasil. \\ ${ }^{b}$ Instituto Federal de Alagoas, Penedo, 57200000, Brasil. \\ ${ }^{c}$ Universidade de Pernambuco, Petrolina, 56328903, Brasil. \\ d Programa de Pós-graduação em Desenvolvimento e Meio Ambiente, Universidade Federal de Sergipe, São Cristóvão, 49100-00o, \\ Brasil. \\ e Programa de Pós-graduação em Educação Profissional e Tecnológica, Instituto Federal de Sergipe, Aracaju, 49055-260, Brasil. \\ *prof.mariasilene@yahoo.com
}

Recebido: 17 dezembro 2016 / Aceito: 12 janeiro 2017 / Publicado online: 15 fevereiro 2017

\begin{abstract}
Resumo
O saber popular sobre plantas medicinais tem auxiliado estratégias de bioprospecção de espécies e direcionado o estudo para a descoberta de novas atividades farmacológicas. O objetivo desta pesquisa foi identificar espécies medicinais utilizadas contra úlcera gástrica pela comunidade do Povoado Vila Capim, Arapiraca, Alagoas e, avaliar a atividade antiulcerogênica gástrica da espécie mais saliente. Para a seleção dos informantes-chave utilizou-se a técnica da bola de neve e aplicadas entrevistas semiestruturadas. A análise das falas foi pela técnica do Discurso do Sujeito Coletivo. Para os testes farmacológicos foram utilizados ratos Wistar machos no modelo de lesão gástrica aguda induzida por etanol absoluto. A toxicidade aguda foi avaliada. Dezessete informantes-chave indicaram os sintomas e plantas medicinais utilizadas contra a úlcera gástrica. A espécie com maior valor de saliência foi a Solanum stipulaceum (sacatinga), escolhida para testes químicos e farmacológicos. O extrato aquoso bruto (SSaq) apresentou atividade significativa na dose de $200 \mathrm{mg} \mathrm{kg}^{-1}(\mathrm{p}<0,05)$, sendo capaz de inibir lesões $(91,35 \%)$. $\mathrm{O}$ extrato etanólico (SSet) demonstrou atividade gastroprotetora nas doses de 200 e $400 \mathrm{mg} \mathrm{kg}^{-1}$ ( $\mathrm{p}<0.01$ ), com inibição de $71,12 \%$ e 76,38\%, respectivamente. É necessário aprofundar os estudos para a elucidação dos compostos químicos das folhas da espécie, toxicologia e identificação dos mecanismos de ação subjacentes à atividade gastroprotetora de S. stipulaceum.
\end{abstract}

Palavras-chave: Plantas medicinais, bioprospecção, análise de saliência, ensaios in vivo.

\section{Applied ethnobotany to gastric ulcer and pharmacological evaluation of Solanum stipulaceum}

\begin{abstract}
The popular knowledge about medicinal plants has helped the bioprospecting strategies of species and directed to a discovery of new pharmacological activities. The aim of this study was to identify medicinal species used against gastric ulcer in the Vila Capim village, Arapiraca, Alagoas, and it evaluating a gastric anti - inflammatory activity of the most salient species. For a selection of key informants has used a snowball technique. The TCLE was signed and semi-structured interviews were applied. The analysis of the speeches was done on the Discourse of the Collective Subject technique. For pharmacological experimental tests, plant extract was underwent the Acute Gastric Lesion Model induced by absolute ethanol and using Wistar male rats. Acute toxicity was evaluated. Seventeen key informants indicated the symptoms and the medicinal plants used against gastric ulcer. The species with the highest salience value was Solanum stipulaceum Roem \& Schult (sacatinga), chosen for chemical and pharmacological tests. The crude aqueous extract (SSaq) presented significant activity at a dose of $200 \mathrm{mg} \mathrm{kg}^{-1}(\mathrm{p}<0.05)$, being able to inhibit lesions (91.35\%). Ethanolic extract (SSet) showed gastroprotective activity at doses of 200 and $400 \mathrm{mg} \mathrm{kg}$ ${ }^{-1}$ ( $\left.\mathrm{p}<0.01\right)$, with inhibition of $(71.12 \%$ and $76.38 \%$ ), respectively. Further studies will be need for analysis of toxicity and identification of the mechanisms of action underlying the gastrointestinal activity of $S$. stipulaceum.
\end{abstract}

Keywords: Medicinal plants, bioprospecction, salience analysis, in vivo assay. 


\section{Introdução}

As plantas medicinais vêm sendo usadas por diversas populações humanas em todo o mundo. Estima-se que cerca de $80 \%$ da população mundial as utilizem como fonte primária de acesso à saúde (Brasil, 2006). A estratégia de escolha de uma planta para uso, desenvolvida por populações humanas ao longo de gerações, pode ser um fator decisivo para a cura de diversas doenças e tais estratégias podem contribuir fortemente para bioprospecção.

Bioprospecção consiste na seleção de espécies vegetais com o objetivo de buscar novos compostos bioativos para fins comerciais, nesse caso, apresenta quatro abordagens para a seleção de plantas: randômica, quimiotaxonômica, etológica e etnodirigida (Albuquerque e Hanazaki, 2006). De acordo com alguns estudos, a abordagem etnodirigida é muito interessante na seleção de plantas para bioprospecção, pois é embasada nos conceitos da Etnobotânica e Etnofarmacologia, que podem ser úteis na indicação de espécies para futuros estudos farmacológicos (Agra et al., 2008; Silva et al., 2010).

O uso popular de plantas medicinais faz parte da cultura de muitas comunidades brasileiras, que utilizam estas espécies para diferentes finalidades. Pesquisas realizadas por Silva et al. (2006) e Lisboa et al. (2006) identificaram o uso de plantas medicinais contra úlcera gástrica em comunidades dos Estados de Sergipe e Alagoas, respectivamente.

A úlcera gástrica é uma doença do trato gastrintestinal, caracterizada por uma elevada acidez resultando em erosões na mucosa do estômago, aguda ou crônica, acompanhada de processo inflamatório e perda de substâncias e tecido, causando extrema dor e desconforto (Dale e Hayllet, 2010).

Espécies de plantas indicadas pelo uso popular já tiveram sua atividade antiulcerogênica comprovada em testes in vivo, por exemplo, Abarema cochliacarpos (Gomes) Barneby \& Grimes (barbatimão; Silva et al., 2010), Maytenus obtusifolia Mart. (bom-nome; Mota et al., 2008), Croton campestris A. St.-Hill (Júnior et al., 2013), Croton urucurana Baillon bark (urucuana) (Cordeiro et al., 2012), Campomanesia xanthocarpa O. Berg (gabiroba; Markman et al., 2004).

O reconhecimento de que a pesquisa de plantas medicinais, usadas por populações tradicionais representa um adequado enfoque para o desenvolvimento de novas drogas é um grande estímulo para que sejam ampliados os estudos voltados para o tratamento de úlcera gástrica.

Esta pesquisa teve por objetivo realizar o estudo etnobotânico de espécies medicinais popularmente utilizadas contra úlcera gástrica pela comunidade do povoado Vila Capim, Arapiraca, Alagoas.

\section{Material e Métodos}

\section{Área de estudo}

O município de Arapiraca está situado na parte central do estado de Alagoas, conhecida como região do Agreste alagoano, com clima tropical subúmido. Sua flora e fauna são constituídas por uma área de transição entre os biomas Caatinga e Mata Atlântica (IBGE, 2014; Souza e Brito, 2012). Possui uma população estimada em 214.006 habitantes (IBGE, 2014). As principais atividades econômicas do munícipio são agricultura, pecuária e indústria. A área de estudo foi o Povoado Vila Capim localizado na Zona Rural a 7 $\mathrm{Km}$, noroeste do centro de Arapiraca, Alagoas. O Povoado é composto por 1.013 famílias, com renda proveniente principalmente da agricultura familiar. Predomina-se nesta região a cultura do fumo, porém, encontram-se algumas culturas de diversificação como mandioca, algodão e milho. Possui um centro comunitário, uma praça, uma escola de Ensino Fundamental, um posto de saúde, campo de futebol e uma creche.

\section{Conhecimento local e uso de plantas medicinais}

No reconhecimento da comunidade, os pesquisadores foram apresentados aos primeiros informantes por uma das autoras do primeiro levantamento realizado na comunidade, publicado por Lisboa et al. (2006). Para a identificação de todos os informantes-chave foi utilizada a técnica de bola de neve (Bailey, 1994), técnica de amostragem não probabilística e intencional, na qual um informante indica outro.

A amostra foi composta pelos detentores do saber popular sobre plantas medicinais, todas do gênero feminino, sendo garrafeiras, rezadoras, agricultoras e doentes de gastrite ou úlcera, totalizando dezessete informantes. As informações socioeconômicas foram obtidas através da aplicação de um formulário. A todos os sujeitos foram apresentados individualmente às perguntas: Quais as plantas da sua região que a senhor (a) usa para tratar úlcera? Como você sabe que alguém está com úlcera (sintomas)? A primeira buscou saber acerca das plantas utilizadas para o tratamento da úlcera, relacionadas aos sintomas. A segunda teve a finalidade de estimular a fala livre acerca dos "sintomas de úlcera".

Para identificar as espécies mais importantes utilizadas pela comunidade no tratamento de úlcera gástrica foi aplicada a técnica da lista livre. Esta técnica consiste em citar componentes de um determinado domínio cultural na ordem em que eles vêm à mente, representando o conhecimento local e sua variação na comunidade (Quinlan, 2005). Foram aplicadas listas livres sucessivas, obtendo-se informações sobre parte da planta utilizada, tipos de usos, preparos medicinais e locais de coleta.

O material botânico foi coletado com a ajuda dos informantes pelo método de turnê guiada, sendo identificado pelo Herbário MAC do Instituto do Meio Ambiente (IMA) localizado em Maceió, Alagoas.

\section{Preparação dos extratos do material vegetal}

A espécie com maior valor de saliência foi coletada para preparação de extratos. Após a coleta, o material vegetal foi seco em estufa $\left(60^{\circ} \mathrm{C}\right)$ e triturado em moinho de facas. O pó foi submetido a extração a frio, tendo sido separado em duas partes: $199.81 \mathrm{~g}$ foram maceradas com 1 litro de água destilada, 3 vezes, durante 5 dias, e após a secagem obteve-se $36 \mathrm{~g}$ com rendimento de 18,02\%; $89.76 \mathrm{~g}$ foram maceradas com etanol absoluto $(500 \mathrm{~mL})$, por 3 vezes, durante cinco dias. Após a secagem obteve-se 5,82 g, e um rendimento de 6,48\%. Foram preparados o extrato aquoso bruto (SSaq) e o extrato etanólico (SSet). Ao final de cada extração, os extratos foram filtrados em papel de filtro e concentrados à pressão reduzida em rotaevaporador (Cechinel-Filho e Yunes, 2001). 


\section{Toxicidade aguda (DL50)}

Ratos Swiss machos e fêmeas foram divididos em grupos ( $\mathrm{n}=5$ ) como se segue: 1) controle negativo; 2) Grupo Experimental - extrato da planta - $5000 \mathrm{mg} \mathrm{kg}^{-1}$. Após o tratamento os animais foram observados durante 14 dias; no $14^{\circ}$ dia os animais foram sacrificados e os órgãos (coração, fígado, rim e pulmão) foram retirados para a pesagem (Souza Brito, 1994).

\section{Lesão gástrica aguda em ratos induzida por etanol}

Foi utilizado o método descrito por Morimoto et al. (1991). Ratos Wistar machos foram mantidos em jejum durante 16 horas. $\mathrm{O}$ grupo controle positivo foi tratado com lansoprazol (30 mg kg-1), o grupo de controle negativo com solução salina $(\mathrm{NaCl} 0,9 \%)$, Tween 80 a $6 \%\left(10 \mathrm{~mL} \cdot \mathrm{kg}^{-1}\right) \mathrm{e}$ os grupos experimentais com os extratos aquoso e o etanólico em doses de 100, 200 e $400 \mathrm{mg} \mathrm{kg} \mathrm{kg}^{-1}$. Após 1 hora de tratamento, os animais receberam por via oral etanol absoluto (1 mL/animal). Uma hora após a administração do etanol, os animais foram sacrificados e os estômagos removidos e abertos pela grande curvatura e lavados com solução salina para avaliação das lesões. Os estômagos foram fotografados e a área da lesão foi calculada utilizando o programa Bioview 4 (AvSoft, Brasil; Khan, 2004).

Os estudos in vivo foram realizados no Centro de Ciências Biológicas e da Saúde, Laboratório de Farmacologia, da Universidade Federal de Sergipe (UFS). O protocolo experimental foi aprovado pelo Comitê de Ética em Pesquisa Animal da Universidade Federal de Sergipe - CEPA, $\mathbf{n}^{\mathbf{o}}$ 26/2011.

\section{Análise de dados}

Os dados coletados das listas livres foram analisados pela análise de saliência, através do software Antropac 1.0 (Borgatti, 1996), levando-se em consideração a ordem e frequência de citação das espécies na listagem livre de cada informante. Quanto maior o valor de saliência de uma planta significa que ela apareceu em muitas listas livres nas primeiras posições em muitos ordenamentos (Quinlan, 2005).

Os dados referentes aos sintomas reconhecidos localmente sobre a úlcera gástrica foram analisados através do método do Discurso do Sujeito Coletivo - DSC (Lefevre e Lefevre, 2010). Essa metodologia consiste em elaborar uma única fala a partir dos dados qualitativos de natureza verbal, obtidos das respostas às perguntas, que represente o coletivo, sendo fundamentada na teoria da representação social (Jodelet, 2001). Foi realizada a comparação dos discursos dos sintomas retratados pelos informantes com a indicação biomédica presente na literatura. As categorias de úlcera gástrica foram classificadas de acordo com o Código Internacional de Doenças (CID-10).

Os dados dos testes farmacológicos foram expressos como média \pm erro padrão da média. A significância estatística das diferenças entre as médias foi determinada por análise de variância (ANOVA) uma via, seguido do teste de Tukey. O limiar de significância foi estabelecido para $\mathrm{p}<0.05$. O programa estatístico utilizado foi o Graphpad Prisma 5.

\section{Resultados e Discussão}

Dentre os entrevistados, $15(88,2 \%)$ indicaram sintomas para o reconhecimento de úlcera gástrica. Foram reportados os sintomas dor $86,4 \%$, vômito $40 \%$, queimor $33,3 \%$ e ardor 20 $\%$. Uma informante relacionou os sintomas da úlcera com a presença da bactéria Helicobacter pylori, relatando cinco sintomas: vômito, enjoo e mal-estar, correspondendo a 6,6\% das citações. O sintoma “dor" teve 12 citações e "queimor" dez citações de espécies pelas informantes (Tabela 1).

Comparando-se os conceitos analisados pelo DSC com o modelo biomédico descrito na literatura sobre os sintomas de úlcera gástrica, muitos sintomas citados pelos informantes são encontrados na literatura biomédica (Tabela 1). Por exemplo, o sintoma de dor no estômago, reconhecido como uma dor transmitida pela estimulação dos receptores nociceptivos gástricos e por mediadores periféricos, tais como a citocinas, prostaglandinas e prótons $\mathrm{H}^{+}$(Kraychete e Guimarães, 2003). $\mathrm{O}$ vômito também é reconhecido como um sinal crônico da úlcera, tendo como principais receptores estimuladores, a histamina, os muscarínicos, os dopamínicos e alguns da 5hidroxitriptamina (Dale e Hayllet, 2010).

Esse cruzamento de informações populares com a literatura demonstra o rico conhecimento das especialistas sobre a úlcera gástrica ao descrever seus sintomas. Essas informações são valiosas para direcionar pesquisas que foquem avaliar farmacologicamente as espécies indicadas para o tratamento de seus respectivos sintomas, sendo útil para a bioprospecção.

As especialistas locais indicaram 23 espécies de plantas para uso em distúrbios do trato gastrintestinal, das quais 16 espécies foram indicadas especificamente para úlcera gástrica. As espécies mais salientes, com seu nome popular e frequência de citação na listagem livre foram: $S$. stipulaceum Willd. ex Roem. \& Schult, (sacatinga, 0,25\%), S. tuberosum L. (batatinha, 0,22\%), Kalanchoe sp., pra tudo (0,17\%), Schinus terebinthifolia Raddi (aroeira, 0,17\%). Os menores índices de saliência foram para: Lauraceae sp. (louro, 0,04\%), Plectranthus amboinicus (Lour.) Spreng (hortelã grande, 0,03\%) e Citrus limon (L.) Osbeck (limão, 0,03\%; Tabela 2).

A espécie $S$. stipulaceum foi a mais saliente entre os informantes (Figura 1). O gênero Solanum é um dos mais amplos do reino vegetal e o maior da família Solanaceae, com cerca de 1500 espécies e 5000 epítetos (Nee, 1999).

A família Solanaceae foi selecionada como uma das famílias piloto para o início do projeto Flora do Brasil, o qual busca descrever até 2020 a extraordinária diversidade de plantas do país (PBI Solanum Project, 2017).

Muitas espécies do gênero Solanum vêm sendo estudadas devido a suas atividades biológicas, tais como: antiulcerogênica, antibacteriana, antiespasmódica, antioxidante e antimutagênica (Amir e Kumar, 2004).

S. stipulaceum é uma espécie endêmica e nativa do Brasil com distribuição em sete estados do Nordeste (Alagoas, Bahia, Ceará, Paraíba, Pernambuco, Piauí, Sergipe), Centro-oeste (Goiás) e Sudeste (Minas Gerais) nos domínios fitogeográficos Caatinga, Cerrado e Mata Atlântica (Solanum, 2017). Ela é conhecida popularmente na região Nordeste como jurubeba (Silva et al., 2006) e como sacatinga, na comunidade estudada nessa pesquisa. 


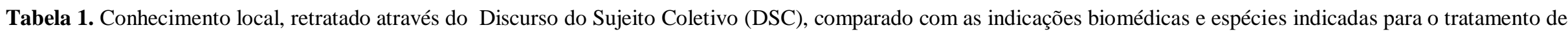
úlcera gástrica pelos informantes do Povoado Vila Capim, Arapiraca - AL, Nordeste do Brasil.

\begin{tabular}{|c|c|c|c|c|}
\hline $\begin{array}{l}\text { Sintomas de } \\
\text { úlcera gástrica }\end{array}$ & DSC & $\begin{array}{l}\text { Citação } \\
(\%)\end{array}$ & Indicação biomédica & $\begin{array}{l}\text { Nome popular das espécies } \\
\text { usadas no tratamento }\end{array}$ \\
\hline Dor & $\begin{array}{l}\text { "Sinto muita dor no estômago - } \\
\text { dores finas, quando como certos } \\
\text { tipos de alimentos como farinha } \\
\text { ou comidas quentes ou geladas a } \\
\text { dor aumenta." }\end{array}$ & $\begin{array}{l}86,7 \% \\
\mathrm{n}=13\end{array}$ & $\begin{array}{c}\text { A dor gastrintestinal é ocasionada por uma grande quantidade de } \\
\text { mediadores periféricos (bradicinina, citocinas, prostaglandinas, } \\
\text { serotonina, ATP, prótons H+) que agem diretamente nos receptores } \\
\text { nociceptivos gastrintestinais iniciando a transmissão dolorosa } \\
\text { (Kraychete e Guimarães, 2003). }\end{array}$ & $\begin{array}{l}\text { Batatinha, laranjeira braba, } \\
\text { barbatimão, hortelã pequena, } \\
\text { sambacaitá, couve, pra tudo, } \\
\text { babosa, sacatinga, alfavaca, } \\
\text { cajueiro roxo e hortelã grande. }\end{array}$ \\
\hline Vômito & $\begin{array}{l}\text { "Tenho crises de vômito quando } \\
\text { vou me alimentar e algumas } \\
\text { vezes vêm acompanhadas de } \\
\text { sangue." }\end{array}$ & $\begin{array}{l}40,0 \% \\
\mathrm{n}=6\end{array}$ & $\begin{array}{l}\text { O vômito é um dos sinais crônicos da úlcera, que compreende a } \\
\text { atividade de coordenação dos músculos involuntários do trato GI e } \\
\text { dos músculos somáticos abdominais e respiratórios, os principais } \\
\text { receptores envolvidos no controle do vômito incluem os H1 da } \\
\text { histamina, os muscarínicos, D2 da dopamina e alguns da 5- } \\
\text { hidroxitriptamina (5-HT3) (Dale e Hayllet, 2010). }\end{array}$ & $\begin{array}{l}\text { Barbatimão, hortelã pequena, } \\
\text { sambacaitá, batatinha, couve, } \\
\text { laranjeira braba, pra tudo e } \\
\text { sacatinga. }\end{array}$ \\
\hline Queimor & $\begin{array}{l}\text { "Sinto uma constante quentura, } \\
\text { uma queimação no estômago." }\end{array}$ & $\begin{array}{c}33,3 \% \\
\mathrm{n}=5\end{array}$ & $\begin{array}{l}\text { Queimação epigástrica - sensação incômoda de calor no epigástrico } \\
\text { (Nadir et. al., 2003). }\end{array}$ & $\begin{array}{l}\text { Batatinha, laranjeira braba, } \\
\text { couve, pra tudo, babosa, } \\
\text { sambacaitá, alfavaca, cajueiro } \\
\text { roxo, hortelã grande e hortelã } \\
\text { pequena. }\end{array}$ \\
\hline $\begin{array}{l}\text { Ardor } \\
\text { Obs*o informante } \\
\text { sempre indicou } \\
\text { este sintoma junto } \\
\text { com o de vômito. }\end{array}$ & $\begin{array}{l}\text { "Noto meu estômago ardendo } \\
\text { muito, principalmente depois que } \\
\text { eu como comida quente." }\end{array}$ & $\begin{array}{c}20,0 \% \\
\mathrm{n}=3\end{array}$ & $\begin{array}{l}\text { Pirose comumente referida como ardor ou queimação, é menos } \\
\text { frequente que a dor esofagiana. } \\
\text { Pirose ou azia a sensação de ardência ou } \\
\text { queimação com início na região epigástrica e/ou retroesternal } \\
\text { (Meyer et al., 2006; Nadir et. al., 2003). }\end{array}$ & Sacatinga. \\
\hline $\begin{array}{l}\text { Dor } \\
\text { Inchaço } \\
\text { Vômito } \\
\text { Enjoo } \\
\text { Mal estar }\end{array}$ & $\begin{array}{l}\text { "Essa bactéria me faz sentir dor } \\
\text { muito forte no estômago, inchaço, } \\
\text { muita vontade de vomitar enjoo e } \\
\text { mal estar quando sinto o cheiro } \\
\text { da comida." }\end{array}$ & $\begin{array}{l}6,7 \% \\
\mathrm{n}=1\end{array}$ & $\begin{array}{l}\text { Dispepsia vem grego pepsis = “digestão", associada ao prefixo } \\
\text { "dis" que significa "digestão alterada". Os sintomas podem incluir } \\
\text { dor epigástrica, pirose retroesternal, azia, sensação de regurgitação, } \\
\text { empachamento, saciedade precoce, náuseas, vômitos, eructação, } \\
\text { entre outros (Silva, 2008). }\end{array}$ & Limão. \\
\hline
\end{tabular}




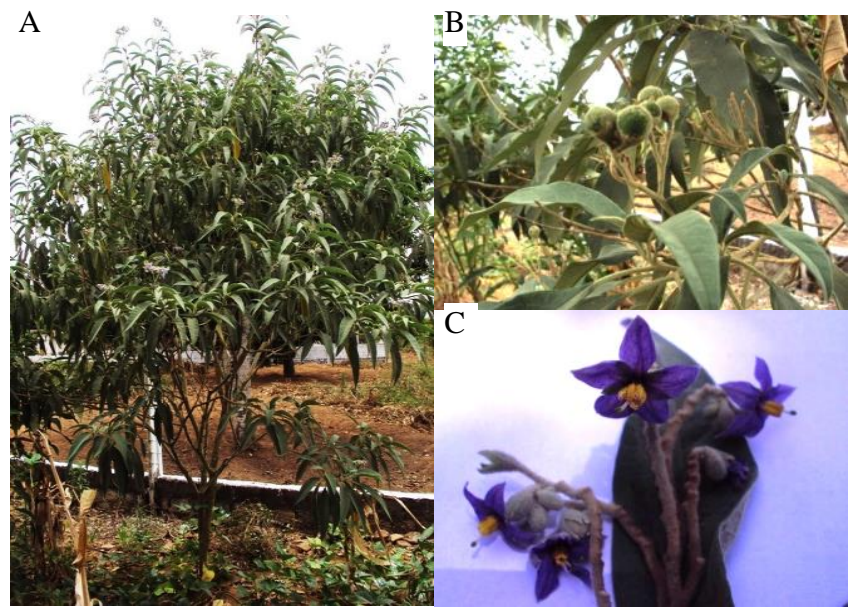

Figura 1. A) Espécie Solanum stipulaceum, cultivada em quintal por informante do Povoado Vila Capim, Arapiraca, Alagoas; B) Folha e fruto de S. stipulaceum; C) Flor de $S$. stipulaceum.

Sendo a espécie mais saliente neste trabalho, procedemos ao estudo in vivo da propriedade antiulcerogênica atribuída pela comunidade.

A toxicidade aguda do extrato aquoso e do etanólico das folhas de $S$. stipulaceum foram testados em camundongos Swiss na dose de $5000 \mathrm{mg} \mathrm{kg}^{-1}$ e não produziram quaisquer sinais ou sintomas visíveis de toxicidade nos animais tratados. Após 14 dias da administração, nenhum animal morreu e nenhuma variação significativa no peso corporal foi observada, como também no peso dos órgãos. Uma vez que não foram observados sinais de toxicidade aguda, seguiu-se a avaliação da atividade gastroprotetora.

O SSaq apresentou atividade gastroprotetora na dose de $200 \mathrm{mg} \mathrm{kg}^{-1}$ (Tabela 2; Figura 2A), sendo capaz de inibir quase que completamente as lesões $(91,35 \%)$, em relação ao controle negativo.

Tabela 2. Atividade antiulcerogênica do extrato aquoso (SSaq) das folhas de Solanum stipulaceum na lesão gástrica, em ratos, induzida por etanol

\begin{tabular}{cccc}
\hline Tratamento & $\begin{array}{c}\text { Dose } \\
\mathrm{mg} \mathrm{kg}^{-1}\end{array}$ & $\begin{array}{c}\text { UI } \\
\mathrm{mm}^{2}\end{array}$ & $\begin{array}{c}\text { Inibição da } \\
\text { úlcera }(\%)\end{array}$ \\
\hline Salina & - & $118,5 \pm 33,0$ & - \\
Lansoprazol & 30 & $18,85 \pm 5,9^{*}$ & 77,7 \\
& 100 & $192,9 \pm 40,1$ & 0,0 \\
SSaq & 200 & $10,2 \pm 4,2^{*}$ & 91,3 \\
& 400 & $50,1 \pm 21,0$ & 57,6
\end{tabular}

Média \pm erro padrão. $* \mathrm{p}<0.05 \mathrm{e} * * \mathrm{p}<0,01$, comparado com o controle pelo teste ANOVA. UI = Índice de Úlcera.

Quando comparado com a droga de referência, lansoprazol, na dose de $30 \mathrm{mg} \mathrm{kg}^{-1}$, o SSaq na dose de 200 $\mathrm{mg} \mathrm{kg}^{-1}$ reportou uma inibição significativa das lesões induzidas pelo etanol absoluto.

O SSet, testado nas doses de 100,200 e $400 \mathrm{mg} \mathrm{kg}^{-1}$ apresentou atividade gastroprotetora nas doses de 200 e 400 $\mathrm{mg} \mathrm{kg}^{-1}(\mathrm{p}<0.01)$ com porcentagem de inibição de $71,1 \% \mathrm{e}$ $76,3 \%$, respectivamente, semelhante ao lansoprazol, que foi de 78,2\% (Tabela 3; Figura 2A e 2B). Estes dados sugerem que o mecanismo antiulcerogênico da espécie $S$. stipulaceum pode estar relacionado à atividade protetora contra ácido gástrico, como faz o lansoprazol ou a um processo de citoproteção.

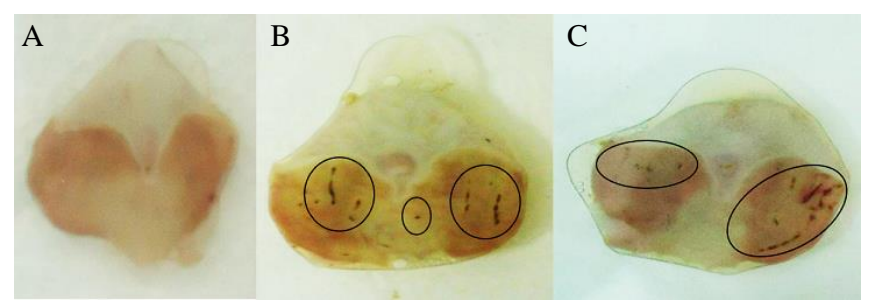

Figura 2. Estômago de rato ulcerado tratado com extrato de Solanum stipulaceum nas seguintes condições: A: extrato aquoso bruto (200 $\mathrm{mg} \mathrm{kg}^{-1}$ ); extrato etanólico na concentração de $200 \mathrm{mg} \mathrm{kg}^{-1}$ (B) e $400 \mathrm{mg} \mathrm{kg}^{-1}$ (C).

Tabela 3. Atividade antiulcerogênica do extrato etanólico (SSet) das folhas de Solanum stipulaceum na lesão gástrica, em ratos, induzida por etanol.

\begin{tabular}{cccc}
\hline Tratamento & $\begin{array}{c}\text { Dose } \\
\mathrm{mg} \mathrm{kg}^{-1}\end{array}$ & $\begin{array}{c}\text { UI } \\
\mathrm{mm}^{2}\end{array}$ & $\begin{array}{c}\text { Inibição da } \\
\text { úlcera (\%) }\end{array}$ \\
\hline Controle & ---- & $181,5 \pm 28,6$ & - \\
Lansoprazol & 30 & $32,9 \pm 2,2^{* *}$ & $78,2 \%$ \\
& 100 & $131,5 \pm 37,1$ & $27,5 \%$ \\
SSet & 200 & $52,4 \pm 19,4^{* * *}$ & $71,1 \%$ \\
& 400 & $42,8 \pm 16,3^{* * *}$ & $76,3 \%$ \\
\hline
\end{tabular}

Média \pm erro padrão. ${ }^{*} \mathrm{p}<0.05 \mathrm{e} * * \mathrm{p}<0,01$, comparado com o controle pelo teste ANOVA. UI = Índice de Úlcera.

Observou-se que o SSaq, na dose de $400 \mathrm{mg} \mathrm{kg}^{-1}$, não apresentou diferença estatística em relação ao controle, apresentando-a apenas no extrato etanólico. Isto pode ser explicado pelas diferenças nos processos extrativos, resultando na seletividade de substâncias em cada extrato. Extratos vegetais brutos constituem uma matriz complexa composta por diversas classes de produtos naturais, contendo diferentes grupos funcionais.

Uma caracterização histoquímica das folhas de $S$. stipulaceum realizada por Matias et al. (2016) identificou a presença de terpenoides, alcaloides, compostos fenólicos, taninos e flavonoides. Lima (2015) realizou uma prospecção fitoquímica das folhas desta espécie por métodos colorimétricos qualitativos e quantitativos e por cromatografia líquida de alta eficiência, detectando fenóis, taninos, esteroides livres, triterpenos, alcaloides, saponinas e flavonoides. Especificamente para o extrato aquoso das folhas, identificouse a presença de derivados do ácido gálico, flavonoides $\mathrm{e}$ terpenos. E para o extrato hidroetanólico foram identificados fenóis, esteroides livres, triterpenoides pentacíclicos, alcaloides, flavonóis, flavononóis e xantonas.

Diversos estudos sobre atividade biológica de plantas medicinais contendo taninos e flavonoides evidenciaram dentre outras ações a cura de feridas, atividades antiinflamatória, antiulcerogênica gástrica e antioxidante (Kushima et al., 2009; Moleiro et al., 2009). Taninos podem interagir com as proteínas do muco, melhorando seu efeito citoprotetor pela formação de um revestimento proteico na mucosa gastrintestinal (Okuda, 2005; Moleiro et al., 2009). 
Porém estes efeitos protetores dependem da dose e do tipo de tanino. Donatini et al. (2009), estudando o efeito gastroprotetor do extrato de Syzygium jambos, cujos princípios ativos nas folhas são flavonóides, taninos e óleos voláteis observaram que, nas doses testadas, no modelo agudo de indução de úlcera houve redução significativa das lesões gástricas, porém quando testado no modelo de úlcera subcrônica o efeito protetor não foi observado.

A atividade antioxidante de compostos fenólicos tem sido mostrada por vários estudos (Borrelli \& Izzo, 2000; Carbonezi et al., 2007; Havsteen, 2002). Taninos são potentes sequestradores de radical peroxil (Okuda, 2005). Esta propriedade antioxidante auxilia no processo de cura, já que os radicais livres são um fator importante na formação de lesões ulcerativas e erosivas do trato gastrintestinal. Nascimento (2006) fez o estudo químico de frações do extrato benzeno:etéreo das folhas de S. stipulaceum detectou a presença de flavonoides, como a quercetina e o kanferol. Por sua vez, Cao et al. (1997) e Arora et al. (1998) destacam que extratos de plantas podem agir como antioxidantes ou próoxidantes, dependendo da estrutura e composição das diferentes classes de polifenóis presentes no extrato e da interação entre estes polifenóis.

Outra classe de compostos importantes detectada nas folhas de $S$. stipulaceum são os terpenos. A atividade antiulcerogênica de triterpenos pentaciclícos foi relatada por Andrade et al. (2008). Uma análise estrutural e histoquímica das folhas de S. stipulaceum revelou a presença de terpenos no parênquima foliar, bem como pronunciado acúmulo destes compostos em quase todos os órgãos desta espécie, indicando uma maior diferença estrutural em relação a outras espécies do gênero (Matias et al., 2016). Alguns constituintes desse grupo, os sesquiterpenos já foram estudados como agentes protetores contra úlcera gástrica, os quais demonstraram significativa proteção contra úlceras induzidas por diferentes modelos clássicos de indução de úlcera. Almeida (2005) ao avaliar a atividade antissecretora da fração semipurificada das folhas de Arctium Lapa L., cujo constituinte majoritário é a onopordopicrina, através de ensaios de somastotatina e gastrina, observou aumento na liberação e/ou produção de somatostatina e diminuição de gastrina.

Diferentes atividades dos extratos de S. stipulaceum têm sido descritas na literatura, porém poucas propriedades medicinais desta espécie são conhecidas ou foram submetidas a estudos farmacológicos. A atividade moluscicida contra Biomphalaria glabrata foi estudada por Silva et al. (2005) utilizando-se o extrato metanólico das raízes e por Silva et al. (2006), testando o extrato alcaloídico de ramos e caule. Os estudos identificaram que tanto o extrato do caule como o da raiz apresentou bioatividade no controle da esquistossomose $($ CL50 $=45,2 \mu \mathrm{g} / \mathrm{mL}$ e CL50 $=48,49 \mu \mathrm{g} / \mathrm{mL}$, respectivamente). Efeito cardiovascular foi relatado para a fração aquosa do extrato etanólico do caule de S. stipulaceum, tendo sido observada hipotensão em ratos de forma dosedependente (Ribeiro et al., 2002).

\section{Conclusões}

Nesta pesquisa, é reportado, pela primeira vez, a ação antiulcerogênica dos extratos aquoso e etanólico das folhas de Solanum stipulaceum, a qual possui substâncias ativas que exercem efeito gastropotetor. Estes resultados confirmam e justificam, ao menos em parte, o uso popular da planta contra distúrbios gastrintestinais. São necessários outros estudos para dar continuidade a elucidação dos compostos fitoquímicos presentes nas folhas de S. stipulaceum, bem como estudos toxicológicos e dos mecanismos de ação subjacentes à sua atividade gastroprotetora.

\section{Agradecimentos}

Aos especialistas locais do Povoado Vila Capim cujo conhecimento popular serviu de base para a realização deste trabalho. À Universidade Estadual de Alagoas - UNEAL, pela ajuda financeira. Ao Conselho Nacional de Desenvolvimento Científico e Tecnológico $(\mathrm{CNPq})$ pelo fornecimento de bolsa à primeira autora.

\section{Referências}

Agra, M.F.; Silva, K.N.; Basílio, I.J.L.D.; Freitas, P.F.; Barbosa-Filho, J.M. 2008. Survey of medicinal plants used in the region Northeast of Brazil. Brazilian Journal of Pharmacognosy, 18(3): 472-508.

Albuquerque, U.P.; Hanazaki, N. 2006. As pesquisas etnodirigidas na descoberta de novos fármacos de interesse médico e farmacêutico: fragilidades e perspectivas. Revista Brasileira de Farmacognosia, 16: 678689.

Almeida, A.B.A. 2005. Atividade antiulcerogênica e antinflamatória intestinal da Arctium lappa. Campinas, SP: [s.n.], Tese (Doutorado) - Universidade Estadual de Campinas. Instituto de Biologia. 167p.

Amir, M.; Kumar, S. 2004. Possible industrial applications of genus Solanum in twenty first century - A review. Journal of Scientific \& Industrial Research, 63(2): 116-124.

Andrade, S. F.; Comunello, E.; Noldin, V. F.; Monache, F. D.; Cechinel Filho, V.; Niero, R. 2008. antiulcerogenic activity of fractions and 3,15-dioxo$21 \alpha$-hydroxy friedelane isolated from Maytenus robusta (Celastraceae). Archives of Pharmacal Research, 31(1): 41-46.

Arora, A., Nair, M.G., Strasburg, G.M., 1998. Structure-activity relationships for antioxidant activities of a series of flavonoids in a liposomal system. Free Radical Biology and Medicine 24, 1355-1363.

Bailey, K. 1994. Methods of Social Research., 4ed. New York: The Free Press, $595 \mathrm{p}$.

Borgatti, S. P. 1996. ANTHROPAC 1.0. NATICK, MA: Analytic Technologies.

Borrelli, F.; Izzo, A.A. 2000. The plant kingdom as a source of anti-ulcer remedies. Phytotherapy Research, 14(8): 1099-1573.

Cao, G., Sofic, E., Prior, R.L. 1997. Antioxidant and prooxidant behavior of flavonoids: structure-activity relationships. Free Radical Biology Medicine, 22, 749-760.

Carbonezi, C.A.; Hamerski, L.; Gunatilaka, A.A.L; Cavalheiro, A.; CastroGamboa, I.; Silva, D.H.S; Furlan, M.; Jovem, M.C.M.; Lopes, M.N.; Bolzani, V.S. 2007. Bioactive flavone dimers from Ouratea multiflora (Ochnaceae). Revista Brasileira Farmacognosia, 17(3): 319-324.

Chechinel-Filho, V.; Yunes, R.A. 2001. Breve análise histórica da Química de Plantas Medicinais: Sua importância na atual concepção de fármaco segundo os paradigmas Ocidental e Oriental. In Plantas medicinais sob a ótica da química medicinal moderna; Yunes, R.A., Calixto, J.B., Eds.; Argos Editora Universitária: Chapecó, Brasil. 500 p.

Cordeiro, K.W.; Pinto, L.A.; Formagio, A.S.N.; Andrade, S.F.; Kassuya, C.A.L.; Freitas, K.C. 2012. Antiulcerogenic Effect of Croton Urucurana Baillon Bark. Journal of Ethnopharmacology, 143(1): 331-337.

Dale, M.M.; Hayllet, D.G. 2010. Farmacologia Condensada. $2^{\text {a }}$ ed. Rio de Janeiro: Elsevier, 136p.

Donatini, R.S.; Ishikawa,T.; Barros, S.B.M.; Bacchi, E.M. 2009. Atividades antiúlcera e antioxidante do extrato de folhas de Syzygium jambos (L.) Alston (Myrtaceae). Revista Brasileira de Farmacognosia, 19(1a): 89-94.

Havsteen, B. 2002. The biochemistry and medical significance of the flavonoids. Pharmacology and Therapeutics 96(2-3): 67-202. 
IBGE - Instituto Brasileiro de Geografia e Estatísticas. 2010. Censo Brasileiro Disponível em: <http://www.censo2010.ibge.gov.br/dados_divulgados>. Acesso em: 30 Mar. 2014.

Jodelet, D. 2001. As representações sociais. In: Representações sociais: um domínio em expansão, $1^{\mathrm{a}}$ ed. UDUERJ, Rio de Janeiro, p. 17-44.

Júnior, F.E.; Oliveira, D.R.; Bento, E.B.; Leite, L.H.; Souza, D.O.; Siebra, A.L.; Sampaio, R.S.; Martins, A.O.; Ramos, A.G.; Tintino, S.R.; Lacerda-Neto, L.J.; Figueiredo, P.R.; Oliveira, L.R.; Rodrigues, C.K.; Sales, V.S.; Figueiredo, F.R.; Nascimento, E.P.; Monteiro, A.B.; Amaro, E.N.; Costa, J.G.; Douglas, M.C.H.; Menezes, I.R.; Kerntopf, M.R. 2013. antiulcerogenic activity of the hydroalcoholic extract of leaves of Croton campestris A. St.-Hill in rodents. Evidence Based Complementary and Alternative Medicine, 2013:10p.

Khan, H.A. 2004. Computer-assisted visualization and quantitation of experimental gastric lesions in rats. Journal of Pharmacological and Toxicological Methods. 49(2): 89-95.

Kraychete, D.C.; Guimarães, A.C. 2003. Hiperalgesia Visceral e Dor Abdominal Crônica: Abordagem Diagnóstica e Terapêutica. Revista Brasileira de Anestesiologia. 53(6): 833 - 853.

Kushima, H.; Nishijima, C.M.; Rodrigues, C.M.; Rinaldo, D.; Sassá, M.F.; Bauab, T.M.; Di Stasi, L.C.; Carlos, I.Z.; Souza Brito, A.R.M.; Vilegas, W.; Hiruma-Lima, C.A. 2009. Davilla elliptica and Davilla nitida: gastroprotective, anti-inflammatory immunomodulatory and antiHelicobacter pylori action. Journal of Ethnopharmacology 123(3): 430438.

Lefevre F, Lefevre A.M.C. 2010. Pesquisa de representação social: Um enfoque qualitativo. Brasília. Líber. Série pesquisa v. 20, 224p.

Lima, C.A.A. 2015. Análise fitoquímica e antiulcerogênica dos extratos e frações das folhas de Solanum stipulaceum will ex. Roem \& shult (sacatinga). Dissertação de Mestrado, Universidade Federal de Sergipe, São Cristóvão, Sergipe. 75p.

Lisboa, M.S.; Ferreira, S.M., Silva, M.S. 2006. Uso de plantas medicinais para tratar úlceras e gastrites pela comunidade do Povoado Vila Capim, município de Arapiraca-AL, Nordeste do Brasil. Sitientibus. Série Ciências Biológicas, 6(esp.): 13-20.

Markman, B.E.O; Bacchi, E.M.; Kato, E.T.M. 2004. Antiulcerogenic effects of Campomanesia xanthocarpa. Journal of Ethnopharmacology, 94 (1): 55-7.

Matias, L.J.; Mercadante-Simões, M.O.; Royo, V.A.; Ribeiro, L.M.; Santos, A.C. Fonseca, J.M.S. 2016. Structure and histochemistry of medicinal species of Solanum. Revista Brasileira de Farmacognosia, 26(2), 147160.

Meyer, I.F.; Kaneshima, E.N.; Souza-Kaneshima, A. M. 2006. Alterações no sistema digestivo desencadeadas pelo quadro infeccioso do Trypanosoma Cruzi. Cesumar, 8(1): 11-23.

Moleiro, F.C.; Andreo, M.A.; Santos, R.C.S.; Moraes, T.M.; Rodrigues, C.M.; Carli, C.B.A.; Lopes, F.C.M.; Pellizzon, C.H.; Carlos, I.Z.; Bauabe, T.M.; Wagner Vilegas, W.; Hiruma-Lima, C.A. 2009. Mouriri Elliptica: validation of gastroprotective, healing and anti-helicobacter pylori effects. Journal of Ethnopharmacology, 123(3): 359-368.

Morimoto, Y.; Shimohara, K.; Oshima, S.; Sukamoto, T. 1991. Effects of the new antiulcer agent KB-5492 on experimental gastric mucosal lesions and gastric mucosal defensive factors, as compared to those of teprenone and cimetidine. Japanese Journal Pharmacology, 57(4): 495-505.

Mota, K. S. de L.; Pita, J. C. L. R.; Estevam, E. C.; Medeiros, V.M.; Tavares, J. F.; Agra, M.F.; Diniz, M.F.F.M; Silva, M.S.; Batista, L.M. 2008. Evaluation of the toxicity and antiulcerogenic activity of the ethanol extract of Maytenus obtusifolia Mart. leaves. Revista Brasileira de Farmacognosia, 18(3): 441-446.

Nadir, F.;Costa, J.S.D.; Nader, G.A.; Motta, G.L.C.L. 2003. Prevalência de pirose em Pelotas, RS, Brasil: estudo de base populacional. Arquivos de Gastroenterologia. 40(1): 31-34

Nascimento, R. J.B. Estudo de espécies do gênero Solanum (Solanaceae): quimiotaxonomia e ensaios biológicos. Dissertação de Mestrado. Universidade Federal da Paraíba. João Pessoa, Paraíba. 2006. 103p.

Nee M. 1999. Synopsis of Solanum in the New World. In: Nee M., Symon D.E., Lester R.N., Jessop J.P. Solanaceae IV: Advances in Biology and Utilization. Royal Botanic Gardens, Kew, p. 285-333.

Okuda, T., 2005. Systematics and health effects of chemically distinct tannins in medicinal plants. Phytochemistry, 66(17), 2012-2031.
PBI Solanum Project. Disponível em: 〈http://www.solanaceaesource.org > Acessado em: 08/01/2017.

Quinlan, M. 2005. Considerations for Collecting Freelists in the Field: Examples from Ethobotany. Field Methods, 17(3): 1-16.

Rao, Y. K.; Fang, S. H.; Tzeng, Y. M. 2008. Antiinflammatory Activities of Flavonoids and a Triterpene Caffeate Isolated from Bauhinia variegata. Phytotherapy Research, 22(7): 957-62.

Ribeiro, E. A. N.; Batitucci, M. C. P.; Lima, J. A. T.; Araújo, I. G. A.; Mauad, H.;Medeiros, I. A. 2002. Cardiovascular effects induced by the aqueous fraction of the ethanol extract of the stem of Solanum stipulaceum in rats. Revista Brasileira de Farmacognosia, 12(1): 34-35.

Silva, P.I.R. da. 2008. Tratamento de náuseas e vômitos na grávida por pressão periódica num ponto de acupuntura. Dissertação de mestrado em medicina. COVILHÃ, 60p.

Silva, T.M.S.; Batista, M.M.; Camara, C.A.; Agra, M.F. 2005. Molluscicidal activity of some brazilian Solanum spp. (Solanaceae) against Biomphalaria glabrata. Annals of Tropical Medicine \& Parasitology, 99(4): 419-425.

Silva, T.M.S.; Câmara, C.A.; Agra, M.D. F.; de Carvalho, M.G.; Frana, M. T.; Brandoline, S.V.P.B.; Paschoal, L.D.S.; Braz-Filho, R. 2006. Molluscicidal activity of Solanum species of the Northeast of Brazil on Biomphalaria glabrata. Fitoterapia, 77(6): 449-452.

Silva, T.M.S.; Nascimento, R.J.B.; Batista, M.M.; Agra, M.F.; Camara, C.A. 2007. Brine shrimp bioassay of some species of Solanum from Northestern Brazil. Brazilian Journal of Pharmacognosy, 17(1): 35-38.

Silva, M.S.; Sánchez-Fidalgo, S.; Talero, E.; Cárdeno, A.; Silva, M.A.; Villegas, W.; Souza-Brito, A.R.M.; Lastra, C.A. 2010. Anti-inflammatory intestinal activity of Abarema cochliacarpos (Gomes) Barneby and Grimes in TNBS colitis model. Journal of Ethnopharmacology, 128(2): 467-475.

Solanum in Flora do Brasil 2020. Jardim Botânico do Rio de Janeiro. (http://floradobrasil.jbrj.gov.br/reflora/floradobrasil/FB14865). Acesso em: 08/01/2017.

Souza Brito, A.R.M. 1994. Manual de Ensaios Toxicológicos in vivo. Unicamp: Campinas/SP- Brasil, 116p.

Souza, M.F.; Brito, M.D. 2012. Identificando a Biodiversidade Local: Uma Proposta de Ensino Interligando Estudantes, Tecnologia e Meio Ambiente. Revista Brasileira de Educação e Meio Ambiente, Rio Grande, $7(2): 62-66$. 